prouvé que la méthode de Schmidt se prêtait admirablement au travail en série.

$5^{\circ}$ Il est done naturel qu'un laboratoire, déjà organisé pour travailler par extraction directe, continue à utiliser ce procédé. Mais il semble logique, au fur et à mesure de l'usure ou de la destruction du matériel, de s'équiper progressivement pour travailler selon la méthode Standard internationale, puisqu'en fin de compte, s'il y a poursuites ou désaccord, c'est à elle qu'on doit avoir recours.

$6^{\circ}$ Quant au laboratoire qui se crée, ou bien dans lequel la méthode volumétrique a été employée jusqu'à maintenant, aucun doute possible : la méthode de Schmidt doit être employée exclusivement.

\title{
L'UTILISATION ÉCONOMIQUE DU FROID ARTIFICIEL DANS LA CONSERVATION ET L'AMÉLIORATION DES FROMAGES A PATE PERSILLÉE
}

\author{
par \\ A. MOULIN \\ Ingénieur d'Agriculture
}

Jusqu'à ces dernières années le froid industriel jouait, en industrie laitière, un rôle assez effacé se bornant à la conservation des dérivés issus de la matière grasse du lait : crème et beurre. Son application assez récente dans l'industrie fromagère, notamment dans l'affinage du Roquefort, laisse apparaître d'heureuses perspectives. D'ores et déjà, la réfrigération peut être considérée comme le complément indispensable de l'affinage puisqu'elle permet de fixer conditionnellement la maturation au point préféré par la clientèle. Au dire de certains techniciens en la matière, les conditions essentielles seraient en somme : l'isolation des fromages du contact de l'air, secondée par un choix judicieux des produits à conserver, l'abaissement de la température du frigorifère au voisinage de $0^{\circ}$ C. et ce, durant au moins six mois. Certes, ces facteurs conditionnent favorablement la réussite mais, à mon avis, reposent sur une théorie par trop étroite et présentent ainsi, dans la pratique, l'inconvénient d'entraîner des dépenses notables par suite du séjour prolongé des fromages dans la chambre frigorifique, à une température excessivement basse, d'où un stockage forcé se traduisant finalement par une immobilisation de capitaux et des frais de conservation supplémentaires.

Deux essais, effectués à l'entrepôt régional de Marseille d'une importante société d'alimentation et portant sur des Bleus d'Auvergne, m'ont démontré qu'il est possible d'obtenir d'excellents 
résultats, tant dans l'amélioration que dans la conservation des produits, tout en s'écartant d'une règle trop rigoureuse quant à son application industrielle.

La première expérience fut tentée du $1{ }^{\text {er }}$ au 30 septembre 1938 ; la deuxième, dura du $1^{\text {er }}$ au 30 avril 1939 ; done similitude quant à la durée de l'épreuve. Les Bleus expérimentés présentaient, dans les deux cas, un épiderme recouvert d'une couche glaireuse de teinte rougeâtre, indice d'une maturation avancée.

Dans le deuxième essai expérimental, afin d'obtenir confirmation, aussi exactement que possible, du premier résultat, le mode opératoire n'était pas modifié. De même, la température de l'air ambiant ne subissait pas de variation thermique notable.

Les fromages, au nombre de 48 , furent pesés individuellement, puis répartis indifféremment en deux lots, appelons-les A et B. Les fromages du lot A furent enveloppés soigneusement dans des feuilles d'étain ; tandis que ceux du lot B étaient abandonnés directement à l'air. La température de la salle de réfrigération oscillait entre $+4^{\circ} \mathrm{C}$. et $+6^{\circ} \mathrm{C}$. et son degré hygrométrique restait stationnaire au voisinage de 90 .

Le mois de séjour en frigorifère écoulé, les fromages furent examinés. Le lot A présentait une croûte de teinte blanchâtre et d'un goût se rapprochant sensiblement du noyau du fromage, lequel avait acquis une pâte onctueuse et d'une saveur remarquablement douce. Le poids, sur l'ensemble des fromages, n'avait pas varié. Par contre, les fromages constituant le lot B avaient subi une nette diminution de poids se traduisant par un déchet représentant approximativement le vingtième du poids global. A l'encontre du lot $\mathrm{A}$, leurs défauts s'étaient aggravés : la pâte était devenue particulièrement friable et la croûte, immangeable d'ailleurs, avait pris une couleur terne. Ces caractéristiques se retrouvèrent encore plus prononcées dans la deuxième expérimentation où les fromages du lot $A$ accusaient une perte de poids légèrement supérieure au vingtième constaté lors de la première épreuve.

Comment se manifeste l'action du froid.

Pour bien comprendre cette discordance dans les résultats, née de l'utilisation ou non d'une simple feuille d'étain, il importe de connaitre par quel jeu de fermentations l'action du froid est-elle efficace ou nuisible?

La maturation des fromages en cave d'affinage s'accomplit sous l'influence combinée de deux natures de fermentations :

10 Fermentations intérieures, se produisant à l'abri de l'air (anaérobies) et jouant, de ce fait, un rôle prépondérant.

$2^{\circ}$ Fermentations extérieures, ayant besoin d'air pour se 
maintenir (aérobies); celles-ci favorisent la formation de la croûte à la surface du fromage.

Par le froid intense, sous l'enveloppe d'étain, les premières sont ralenties, tandis que les secondes, dépourvues d'air, sont complètement enrayées. Il en résulte que la croûte déjà formée sur le fromage tend à disparaître par voie de transformation, car, malgré un abaissement notable de la température ambiante, les fermentations anaérobies se produisent encore et déterminent des réactions accompagnées d'un dégagement de chaleur, et partant d'une élévation de température à l'intérieur. Cet accroissement de température occasionne la vaporisation d'eau et des principes volatils contenus dans le fromage. D'autre part, la température de l'intérieur du fromage étant le plus souvent plus élevée que celle de l'air de la salle, et l'enveloppe d'étain s'opposant à la sortie des éléments constitutifs vaporisés, cette enveloppe va faire office de paroi condensante. S'interposant entre la feuille et la croûte, apparaît le liquide qui, ne pouvant s'écouler au dehors, ramollit la croûte et tend à la rendre de même texture et de même composition que le noyau en l'imprégnant de principes qu'elle avait perdus au cours de l'affinage. A cette action physico-chimique, s'ajoute la privation complète de lumière. Si bien, qu'il suffit d'un mois de froid vif pour retrouver le fromage tendre du centre à la périphérie et beaucoup plus homogène qu'à l'affinage.

La conservation par le froid tend done à homogénéiser la pâte du fromage et, par là même, le rendre entièrement comestible.

Somme toute, grâce à l'emploi judicieux du froid artificiel, le producteur, outre une amélioration qu'il obtient dans la qualité de ses produits, peut stocker afin d'attendre des cours plus avantageux, tout en étant assuré de conserver ses fromages au stade de maturation répondant le mieux au goût du consommateur.

Ces avantages appréciables permettent d'augurer favorablement quant à l'avenir de cette dérivation rationnelle de l'affinage. 